\title{
GaP(001) and InP(001): Reflectance anisotropy and surface geometry
}

\author{
N. Esser \\ Institut für Festkörperphysik, Technische Universität Berlin, Hardenbergstrasse 36, 10623 Berlin, Germany
}

W. G. Schmidt and J. Bernholc

Department of Physics, North Carolina State University, Raleigh, North Carolina 27695-8202

A. M. Frisch, ${ }^{\text {a) }}$ P. Vogt, M. Zorn, M. Pristovsek, and W. Richter

Institut für Festkörperphysik, Technische Universität Berlin, Hardenbergstrasse 36, 10623 Berlin, Germany

F. Bechstedt

Institut für Festkörpertheorie und Theoretische Optik, Friedrich-Schiller-Universität, Max-Wien-Platz, 1, 07743 Jena, Germany

Th. Hannappel and S. Visbeck

Hahn-Meitner Institut, CD, Glienickerstr. 100, 14109 Berlin, Germany

(Received 20 January 1999; accepted 30 March 1999)

\begin{abstract}
We have investigated the optical anisotropy of $\mathrm{GaP}(001)$ and $\operatorname{InP}(001)$ surfaces. The samples were prepared by homoepitaxial metalorganic vapor phase epitaxy growth and either directly transferred into ultrahigh vacuum (UHV) or in situ capped and, after transfer, decapped in UHV by thermal desorption of a P/As capping layer. Symmetry, composition, and surface optical anisotropy were characterized by low-energy electron diffraction, Auger electron spectroscopy, and reflectance anisotropy spectroscopy. We observe $(2 \times 1) /(2 \times 2)$-like reconstructions for the very P-rich and $(2 \times 4)$ reconstructions for the more cation-rich surfaces. No $(4 \times 2)$ reconstruction could be prepared, independent of the preparation method. A comparison of the reflectance anisotropy between $\mathrm{GaP}(001)$ and $\operatorname{InP}(001)$ surfaces shows similar line shapes for the very cation-rich $(2 \times 4)$ surfaces. For less cation-rich surfaces, however, we observe distinct differences between the spectra of the two systems. In both cases, different line shapes in the reflection anisotropy spectra occur for the $(2 \times 4)$ periodicity, suggesting the existence of different $(2 \times 4)$ geometries. The experimental results are discussed on the background of atomic structures, total energies and reflectance anisotropy spectra obtained ab initio from density-functional theory local-density approximation calculations. () 1999 American Vacuum Society. [S0734-211X(99)03504-0]
\end{abstract}

\section{INTRODUCTION}

Much progress has been made in recent years in understanding the microscopic structure of the growth planes of III-V compound semiconductors (for a recent review, see, e.g., Ref. 1). Optical anisotropy at the same time has been proven to be an additional and complementary tool to electron spectroscopic methods for investigating the microscopic surface structure of semiconductors. ${ }^{2,3}$ Previous investigations of $\operatorname{GaAs}(001)^{4,5}$ and $\operatorname{InP}(001)^{6}$ surfaces have shown that the atomic structure can be successfully clarified in many cases by comparing total energy and band structure calculations for the optical anisotropy on the one hand with experimental data of the optical anisotropy on the other hand. The atomic surface structures of the $(2 \times 4)-\mathrm{GaP}(001)$ and $-\operatorname{InP}(001)$ surfaces were recently investigated by density functional theory local-density approximation (DFT-LDA) total energy calculations. ${ }^{7,8}$ As in the case of $\operatorname{InP}(001)$, the atomic structures of $\mathrm{GaP}(001)$ were found to be different from the well-investigated $\mathrm{GaAs}(001)$ surface, ${ }^{9-11}$ due to a large size difference between cations and anions.

While several recent studies have addressed the atomic structure of $\operatorname{InP}(001),{ }^{8,12-14}$ much less is known about

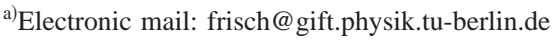

$\mathrm{GaP}(001)$. Available experimental studies lead to different and partially contradictive conclusions about the symmetry and structure of the $\mathrm{GaP}(001)$ surface. So far it has been suggested mostly that ion bombardment and annealing as well as decapping of $\mathrm{GaP}(001)$ results in a $(4 \times 2) / c(8 \times 2)$ reconstructed, Ga-rich surface, ${ }^{15-21}$ in analogy to the corresponding Ga-rich $\mathrm{GaAs}(001)$ surface structure. ${ }^{9}$ In a more recent investigation by ion scattering, ${ }^{22}$ however, a $(2 \times 4)$ symmetry was reported. Similar ambiguous results for $\mathrm{GaP}(001)$ can be found in literature for gas source molecular beam epitaxy (MBE) experiments: In Ref. 23 it is reported that $(2 \times 4)$ and $(4 \times 2)$ reconstruction pattern correspond to $\mathrm{P}$ - and Ga-stabilized surfaces, whereas in Ref. 24 , a $(2 \times 4)$ reconstruction was observed under $\mathrm{Ga}$ as well as under $\mathrm{P}$ supply. Additionally, the authors of Ref. 24 found an intermediate $(4 \times 4)$ reconstruction during layer growth and suggested the formation of $\mathrm{Ga}$ droplets on a $(2 \times 4)$ reconstructed surface for high amounts of Ga supply. Only few data can be found in literature about the optical anisotropy of $\mathrm{GaP}(001)$. To our knowledge, there exist studies of $\mathrm{GaP}(001)$ surfaces in MBE, metalorganic vapor phase epitaxy (MOVPE) and chemical beam epitaxy (CBE). ${ }^{25-28}$ Apart from Ref. 7 no reflectance anisotropy data for $\mathrm{GaP}(001)$ surfaces in ultrahigh vacuum (UHV) are available. In the present study, clean and well-ordered (001) sur- 
faces of MOVPE-grown GaP and InP with different stoichiometries are prepared under ultrahigh vacuum conditions. All samples are characterized by low-energy electron diffraction (LEED), Auger electron spectroscopy (AES), and reflectance anisotropy spectroscopy (RAS). Based on total-energy (TE) calculations and by comparison of experimental data to calculated optical anisotropies for various atomic structure models it is shown that several $(2 \times 4)$ reconstructions exist for $\mathrm{GaP}(001)$ and $\operatorname{InP}(001)$. They realize different stoichiometries. $(4 \times 2)$ structures do not occur in either case.

\section{METHOD}

The $\mathrm{GaP}(001)$ surfaces were prepared by thermal desorption of a protective arsenic/phosphorus double layer (cap) under UHV conditions. For this purpose, homoepitaxial GaP epilayers were grown by MOVPE on highly $n$-doped $\left(10^{18} \mathrm{~cm}^{-3}\right) \mathrm{GaP}(001)$ substrates and capped in situ utilizing the photodecomposition of phosphine and arsine by an excimer laser source. ${ }^{27,29(a)}$ The thermal desorption of the protective arsenic/phosphorus layer was performed at $690 \mathrm{~K}$ in UHV at a base pressure $\leqslant 10^{-10} \mathrm{hPa}$. The $\mathrm{InP}(001)$ samples were grown by MOVPE on $n$-doped $\left(10^{17} \mathrm{~cm}^{-3}\right) \operatorname{InP}(001)$ substrates and transferred under UHV conditions in the analyzing UHV chamber directly after growth, without using the capping technique. ${ }^{29(\mathrm{~b})}$ Starting with P-rich surface conditions in either case, different reconstructions were prepared by subsequent annealing in UHV. After cooling to room temperature, the surfaces were investigated by LEED, RAS, and AES.

The calculations were based on DFT-LDA. Optical properties are calculated in independent-particle approximation from the DFT-LDA electronic structure. To determine the optical anisotropy, we follow the formalism developed by Del Sole ${ }^{30}$ and Manghi et al. ${ }^{31}$ Computational details can be found in Refs. 6 and 11.

\section{RESULTS AND DISCUSSION}

\section{A. Surface symmetry and composition}

The decapped $\mathrm{GaP}(001)$ surfaces (after annealing to 690 K) show a $(2 \times 1) /(2 \times 2)$-like LEED pattern [see Fig. 1(a)], with clear $(2 \times 1)$ spots plus additional streaks in the [110] half order position. A similar LEED pattern [see Fig. 1(b)] is observed from InP(001) surfaces, directly after transfer into the UHV system. Annealing to 785 and $645 \mathrm{~K}$ leads to (2 $\times 4)$ LEED patterns for $\mathrm{GaP}(001)$ as well as for $\operatorname{InP}(001)$. The LEED pattern of the $(2 \times 4) \mathrm{GaP}(001)$ surface does not allow us to distinguish between a $(2 \times 4)$ and a $(2$ $\times 4) / c(2 \times 8)$ reconstruction [Fig. $1(\mathrm{c})]$ due to not fully resolved fractional order spots in the [110] direction. The LEED pattern of the $\operatorname{InP}(001)$ surface, on the contrary, shows a $(2 \times 4)$ periodicity, or a superposition of $(2 \times 1)$ / $(2 \times 2)$ and $(2 \times 4)$ [Fig. $1(d)$ ]. Upon further annealing up to $1000 \mathrm{~K}$ the quality of the LEED pattern for $\mathrm{GaP}(001)$ is improved and the former streaks develop into sharp spots indicating a pure $(2 \times 4)$ reconstruction, similar to the pattern observed for $\operatorname{InP}(001)$ [Figs. 1(e) and 1(f)]. Upon an- (a)

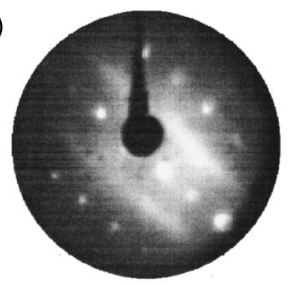

(c)

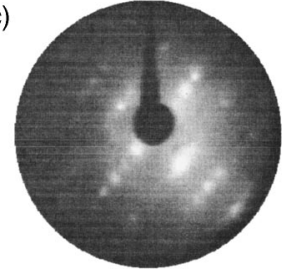

(b)

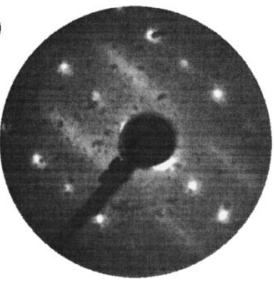

(d)

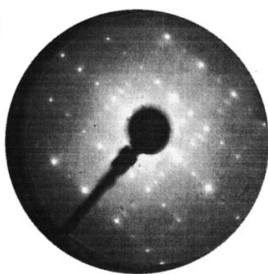

(e)

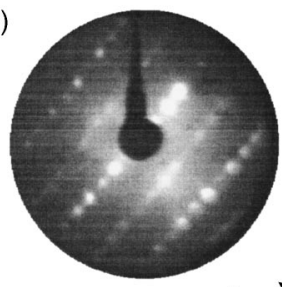

(f)

$[110]$ $[\overline{1} 10]$

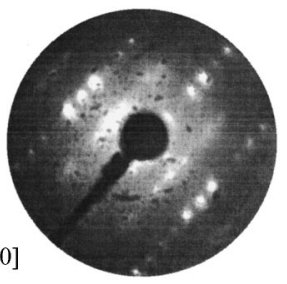

FIG. 1. LEED pattern recorded from $\mathrm{GaP}(001)$ and $\operatorname{InP}(001)$ : (a) $(2 \times 1) /$ $(2 \times 2) \mathrm{GaP}(001)$ taken at a electron energy of $53 \mathrm{eV},($ b) $(2 \times 1) /(2 \times 2)$ $\mathrm{InP}(001)$ (electron energy $50 \mathrm{eV})$, (c) P-rich $(2 \times 4) \mathrm{GaP}(001)$ (electron energy $56 \mathrm{eV})$, (d) $(2 \times 4) \mathrm{InP}(001)$ (electron energy $120 \mathrm{eV})$, (e) Ga-rich $(2 \times 4) \mathrm{GaP}(001)$ (electron energy $52 \mathrm{eV}$ ), (f) $\mathrm{InP}(001)$ (electron energy 50 $\mathrm{eV})$.

nealing to still higher temperatures $[\geqslant 1000 \mathrm{~K}$ for $\mathrm{GaP}(001)$ and $\geqslant 725 \mathrm{~K}$ for $\operatorname{InP}(001)]$ the symmetry of the LEED patterns remains unchanged until the surfaces deteriorate by forming $\mathrm{Ga}$ or In droplets, as indicated by a metallic component in the Ga $3 d /$ In $4 d$ core level photoemission lines (not shown here). AES measurements performed on GaP after decapping and InP after UHV transfer show no detectable carbon or oxygen contaminations. Moreover, the composition of the $\mathrm{GaP}(001)$ surfaces was examined. The P/Ga ratio for the $(2 \times 1) /(2 \times 2)$-like $\mathrm{GaP}(001)$ surface shows P-rich surface conditions, but it cannot be exactly determined because of the $\mathrm{P}$ desorption induced by the electron beam. On the $(2 \times 4)$ reconstructed $\mathrm{GaP}(001)$ surface at low annealing temperatures (only slightly above $785 \mathrm{~K}$ ), the $\mathrm{P} / \mathrm{Ga}$ intensity ratio is close to the one of the $\mathrm{GaP}(110)$ cleavage face (balanced stoichiometry). Upon annealing to higher temperatures $(1000 \mathrm{~K})$ the ratio changes successively by a factor of approximately two towards a Ga-rich surface, suggesting the existence of a more P-rich $(2 \times 4)$ at lower and a Ga-rich $(2 \times 4)$ reconstruction at higher annealing temperatures.

\section{B. Optical anisotropy: Experimental results}

Real parts of different RAS spectra recorded from $\mathrm{GaP}(001)$ and $\operatorname{InP}(001)$ surfaces are shown in Fig. 2. For comparison, a RAS spectrum of the $\beta 2(2 \times 4) \mathrm{GaAs}(001)$ is 
a)

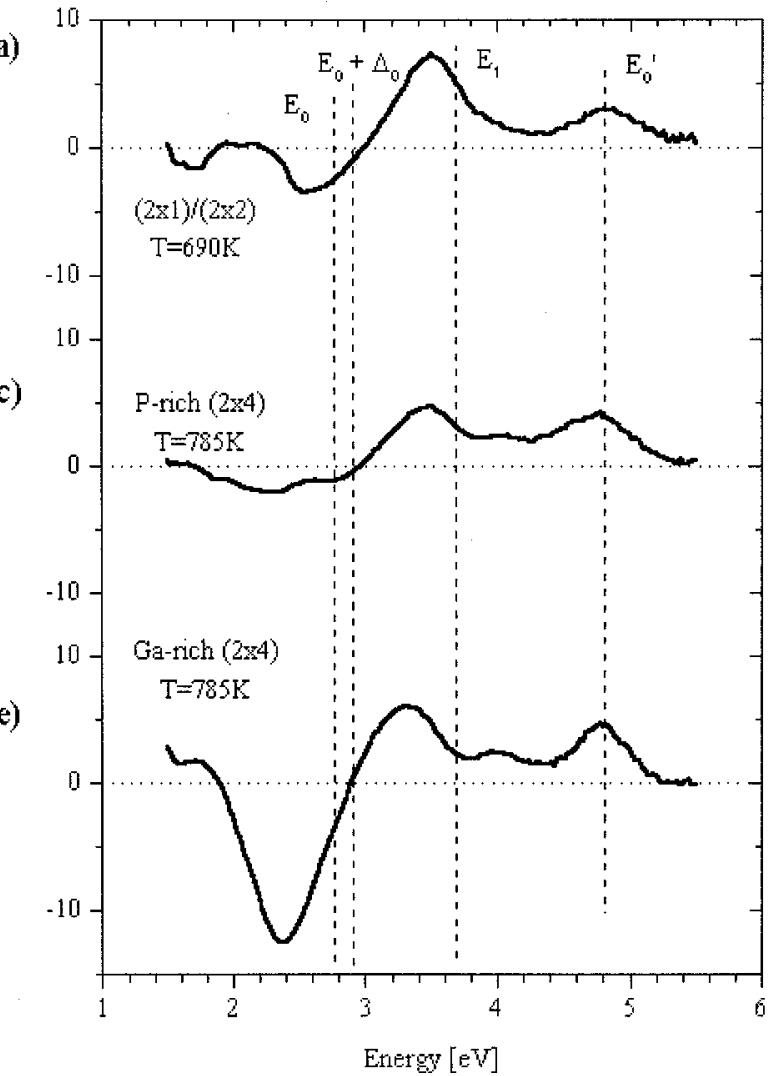

b)

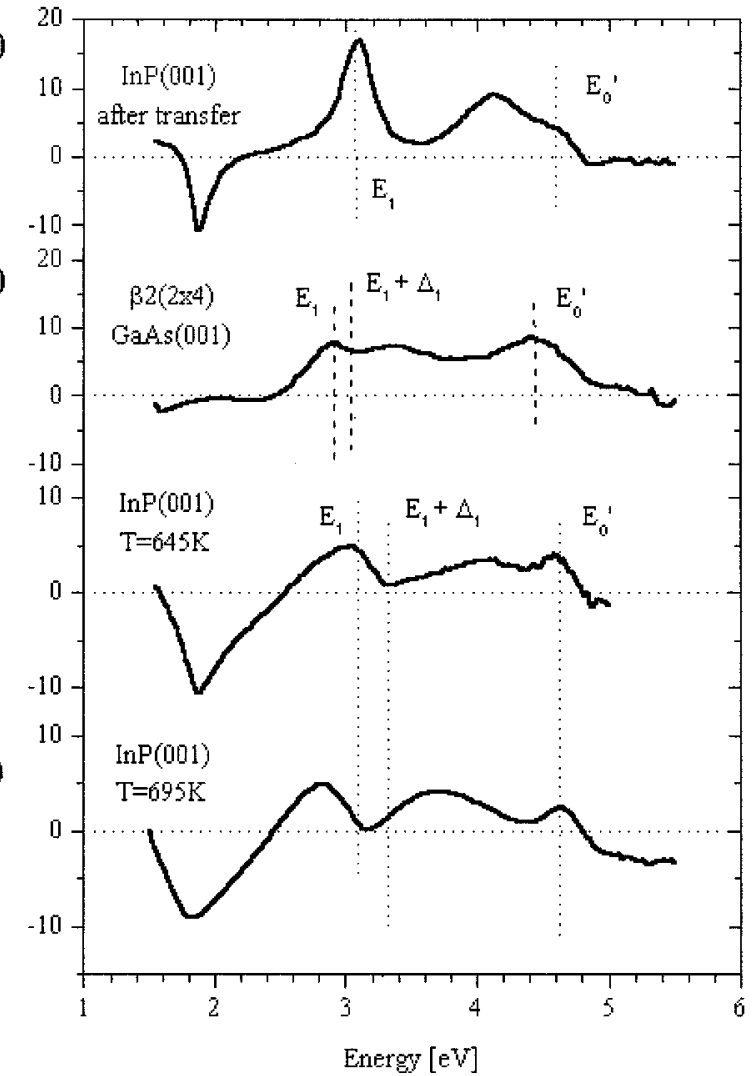

FIG. 2. Real parts of $\left(\left(r_{[110]}^{-} r_{[110]}\right) /\langle r\rangle\right) \times 10^{3}$ (LEED patterns and annealing temperatures are indicated in the plot) for: GaP(001) [left panel, (a), (c), (e)] $\mathrm{InP}(001)$ [right panel, (b), (f), (g)], and $\beta 2(2 \times 4) \mathrm{GaAs}(001)$ [right panel, (d)].

shown in Fig. 2(d). Three different spectral line shapes are reproducibly observed on $\mathrm{GaP}(001)$ and $\mathrm{InP}(001)$, correlated to the surface preparation: a characteristic line shape belongs to the $(2 \times 1) /(2 \times 2)$-like structures [Figs. 2(a), 2(b)], and two different line shapes are observed in either case for $(2$ $\times 4)$ surface structures, one correlated to P-rich conditions at lower annealing temperature [Figs. 2(c), 2(f)] and one to cation-rich conditions at higher annealing temperature [Figs. 2(e), 2(g)].

The P-rich GaP(001) $(2 \times 4)$ surface [Fig. 2(b)] shows a spectrum which is qualitatively similar to the one observed for the As dimer terminated $\beta 2(2 \times 4) \quad \operatorname{GaAs}(001)$ surface. $^{3-5}$ It is dominated by pronounced maxima at 3.5 and $4.8 \mathrm{eV}$, close to the $E_{1}$ and $E_{0}^{\prime}$ gap energies of $\mathrm{GaP}$ (3.69 and $4.77 \mathrm{eV}$, respectively ${ }^{32}$ ) and a weak maximum at $4 \mathrm{eV}$. The RAS spectrum of InP under comparable conditions has a different line shape: In addition to maxima around the bulk critical points $E_{1}$ and $E_{0}^{\prime}\left(3.16\right.$ and $4.68 \mathrm{eV}$, respectively $\left.{ }^{33}\right)$ a strong minimum at $1.9 \mathrm{eV}$ shows up. We mention that in Ref. 34 a somewhat different spectrum (nearly no negative anisotropy) has been assigned to the P-rich phase of InP ( 2 $\times 4)$. The reason for that difference is not clear at present. The line shapes shown in Figs. 2(f) and 2(g) are, however, reproducibly observed in our UHV experiments, whereas a line shape comparable to Ref. 34 could not be obtained in spite of repeated attempts. After annealing to higher temperatures the RAS spectra for $\mathrm{GaP}$ and $\mathrm{InP}(001)$ change and become similar in line shape. New features in the GaP spectrum are a strong minimum at $2.4 \mathrm{eV}$ and a maximum at 3.2 $\mathrm{eV}$, significantly below the $E_{1}$ gap of GaP. On InP the first maximum shifts to somewhat lower energies and an additional maximum appears between $E_{1}$ and $E_{0}^{\prime}$. On both materials, the maximum at the $E_{0}^{\prime}$ point remains unaffected.

Summarizing the experimental results, there are clear similarities between the very anion-rich $(2 \times 1) /(2 \times 2)$-like and the very cation-rich $(2 \times 4)$ reconstructed $\mathrm{GaP}(001)$ and InP $(001)$ surfaces, in particular, if one takes into account the different bulk critical point energies of the two materials. The spectrum of the P-rich $(2 \times 4)$ reconstructed $\mathrm{GaP}(001)$ surface, however, is distinct from the corresponding InP spectrum and resembles the one of $\operatorname{GaAs}(001) \beta 2(2 \times 4)$. The evolution of the RAS spectra of $\mathrm{GaP}(001)$ with increasing $\mathrm{Ga}$ coverage of the surface implies that at least two distinct $(2 \times 4)$ structures with different stoichiometry exist on the $\mathrm{GaP}(001)$ surface. This conclusion cannot be drawn for InP(001) surfaces. Scanning tunneling microscopy images ${ }^{35}$ imply that the transition structure corresponds to a twodomain surface composed of P-rich $(2 \times 1) /(2 \times 2)$ and In-rich $(2 \times 4)$ domains. Also the RAS spectrum [Fig. 2(f)] can be reproduced by a linear superposition of spectra 2(b) and $2(\mathrm{~g})$.

\section{Atomic structure of the surfaces}

So far, only the $(2 \times 4)$-surface atomic structures [especially for $\operatorname{InP}(001)]$ have been analyzed in detail, while the 


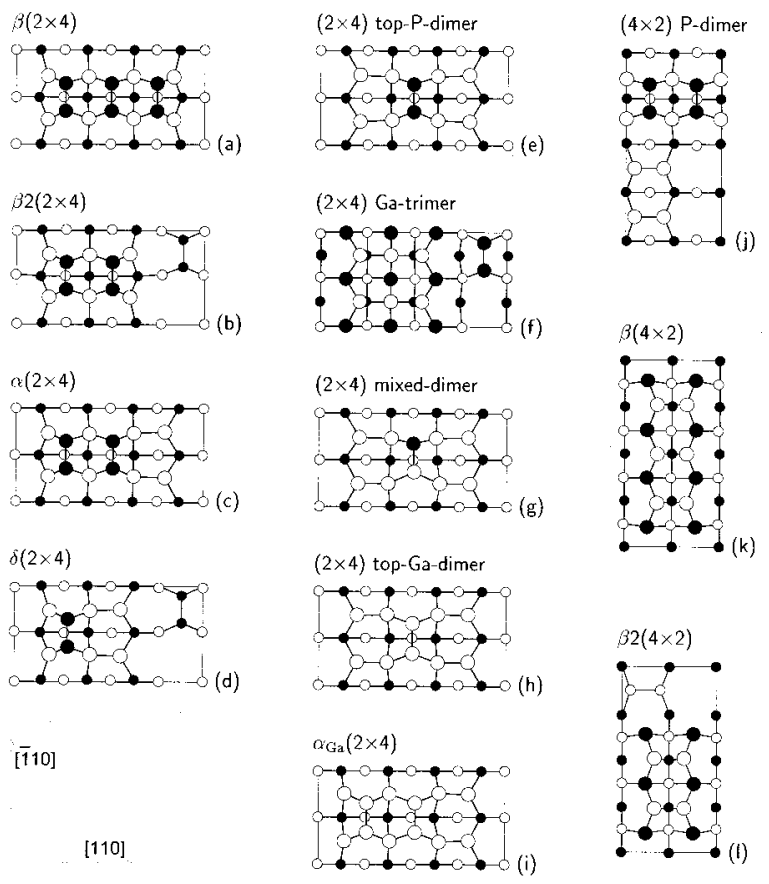

FIG. 3. Top view of relaxed $\mathrm{GaP}(001)(2 \times 4)$ and $(4 \times 2)$ surface reconstruction models. Empty (filled) circles represent $\mathrm{Ga}(\mathrm{P})$ atoms. Large (small) symbols indicate positions in the first and second (third and fourth) atomic layers. $(2 \times 4)$ and $(4 \times 2)$ reconstructions are ordered by increasing Ga coverage.

structure of the $(2 \times 1) /(2 \times 2)$-reconstructed surfaces is still completely unclear. STM and photoemission data are available for $\operatorname{InP}(001)(2 \times 4){ }^{8,13,29(\mathrm{a})} \mathrm{TE}$ calculations performed on InP for a large variety of different $(2 \times 4)$ - and $(4 \times 2)$-reconstruction models revealed a new type of dimer reconstruction involving mixed In-P surface dimers for Inrich surfaces, consistent with the experimental data. ${ }^{8}$ Very recently, TE calculations have also been performed for a series of structural models for $\mathrm{GaP}(001){ }^{7}$ Figure 3 shows the structural models considered for GaP. One of these models, the $\delta(2 \times 4)$ surface structure, which is believed to describe the Sb-induced GaAs $(2 \times 4)$ reconstruction, ${ }^{36}$ turned out to be energetically favored for $\mathrm{GaP}(001)$, but was not considered in earlier studies on $\operatorname{InP}(001)$. We included that model in the present work.

The investigated models realize different In or Ga coverages and can therefore be compared energetically only by taking into account the chemical potentials of the surface constituents. ${ }^{11}$ In Fig. 4 we show the relative formation energies of the considered surface structures vs the cation chemical potential for both $\operatorname{InP}(001)$ and $\mathrm{GaP}(001)$ surfaces. The phase diagrams show following fundamental similarities:

(i) $(2 \times 4)$ structures represent the stable surfaces under cation-rich conditions, in contrast to the $\mathrm{GaAs}(001)$ case where $(4 \times 2)$ structures are known to exist under cation-rich conditions.

(ii) A new atomic surface structure, consisting of mixed cation-P dimers adsorbed on top of a complete cation layer

(a)

P-rich

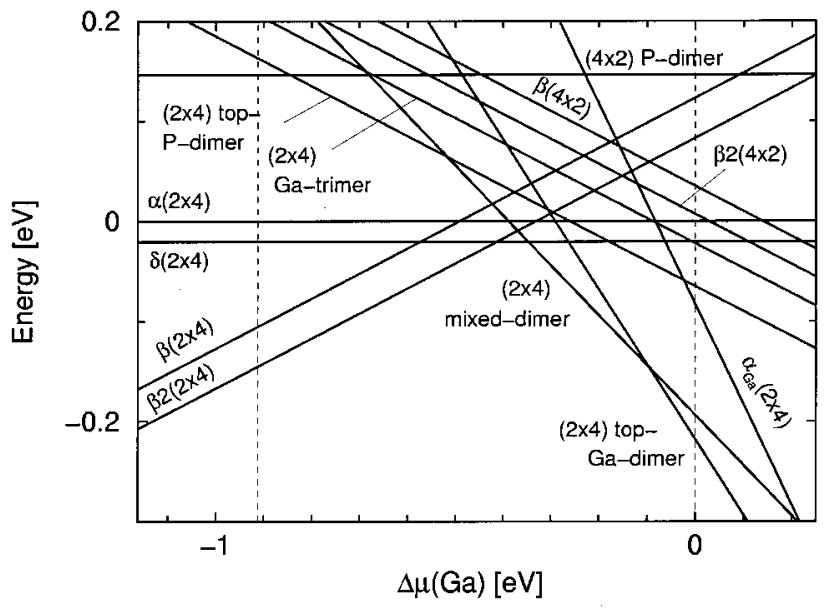

(b)

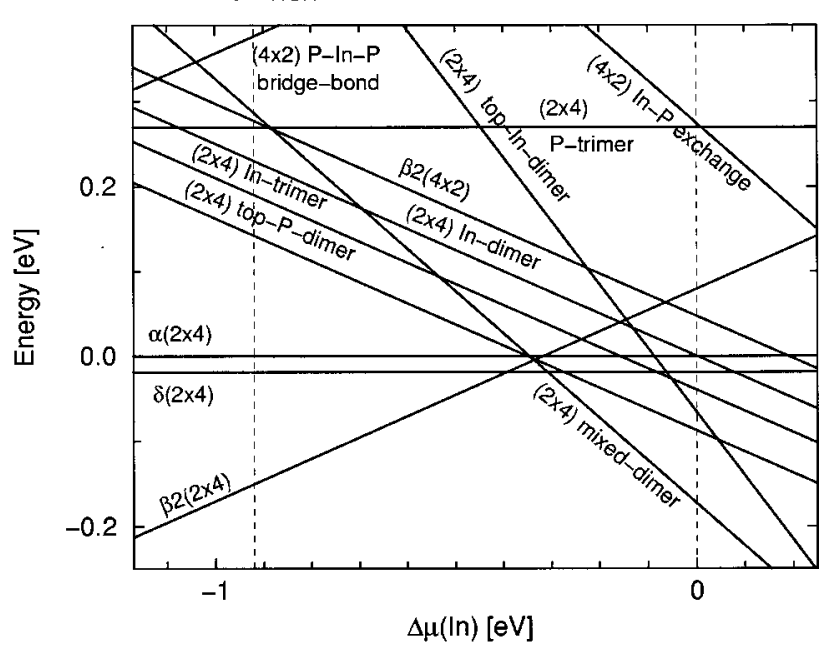

FIG. 4. (a) Relative formation energy per $(1 \times 1)$ unit cell for $\mathrm{GaP}(001)$ surface reconstructions vs $\Delta \mu(\mathrm{Ga}):=\mu_{\text {bulk }}(\mathrm{Ga})-\mu(\mathrm{Ga})$. The approximate thermodynamically allowed range: $\left.-\Delta H_{f}(\mathrm{GaP}) \leqslant \Delta \mu(\mathrm{Ga})\right) \leqslant 0$ is indicated by dashed lines. (b) Same as in (a), but for $\operatorname{InP}(001)$ surface structures.

[Fig. 3(g)] represents the lowest energy structure over a prominent range of chemical potentials.

(iii) For a certain range of preparation conditions, the $\delta$ geometry [Fig. 3(d)] may occur. Given the limited accuracy of our calculations, ${ }^{11}$ further structures such as $\alpha$ and top P dimer [Figs. 3(c) and 3(e)] cannot be excluded for the transition between cation- and anion-rich surfaces. Finally, under P-rich conditions $\mathrm{P}$ dimers in a $\beta 2(2 \times 4)$ geometry [Fig. $3\left(\right.$ b)], as known from As-rich GaAs(001) surfaces, ${ }^{10,37}$ represent the lowest energy structure. However, this result must be taken with caution, since for P-rich conditions the experimental results indicate the appearance of $(2 \times 1) /(2 \times 2)$ structures on both $\mathrm{GaP}$ as well as $\mathrm{InP}$, which were not considered in the TE calculations.

(iv) All equilibrium structures are in agreement with electron counting heuristics. ${ }^{38}$

The similarity between the surface phase diagrams of InP and $\mathrm{GaP}$ points towards a common mechanism: The size 
difference between anions and cations hinders the accommodation of $s p^{2}$-hybridized cation dimer atoms that are typical for cation-rich $\mathrm{GaAs}(001)$ surfaces. Instead, the formation of single-dimer structures is favored for cation-rich surfaces.

Besides these similarities, however, there is also an obvious difference: On $\operatorname{InP}(001)$ the mixed-dimer structure appears to be the only stable geometry under In-rich conditions, while on $\mathrm{GaP}(001)$ the top-Ga dimer model [Fig. 3(h)] is lower in energy in the extreme cation-rich limit.

The phase diagrams of $\mathrm{GaP}$ and $\mathrm{InP}$ in Fig. 4 demonstrate the existence of different stable $(2 \times 4)$ surface phases depending on the value of the cation chemical potential, i.e., the surface preparation conditions. This finding is in excellent agreement with the qualitative evolution of the surface optical anisotropy on $\mathrm{GaP}(001)$.

Since RAS as an optical technique is applicable in UHV as well as gas phase surroundings, the dependence of InP and $\mathrm{GaP}$ surface structures on different environments (e.g., growth conditions in MOVPE, MBE, CBE, and UHV) can be addressed by comparing the RAS spectra taken under different conditions. Comparing our spectra with the ones taken in MOVPE and CBE reveal, that for $\mathrm{InP}$ as well as for $\mathrm{GaP}$, the same line shapes occur in the different environments. ${ }^{26-28,39}$ Moreover, an excellent agreement between symmetry observed by RHEED in $\mathrm{CBE}^{28,39}$ and LEED in our $\mathrm{UHV}$ experiments is found in both $\mathrm{InP}$ and $\mathrm{GaP}$ cases. There is, however, one exception: A $\beta 2(2 \times 4)$ seemingly observed in MBE for $\operatorname{InP}(001)^{34}$ is not showing up in our experiments.

\section{Optical anisotropy: Calculation}

As shown above, the evolution of the surface optical anisotropy and the calculated equilibrium surface structures is qualitatively consistent. An even more direct comparison between theory and measurement, however, is desirable in order to identify specific surface structures. To this end, we compare the computed surface reflectance anisotropy of the energetically favored structures with the experimental RAS spectra.

Simulated spectra for $\mathrm{GaP}(001)$ are shown in the upper panel of Fig. 5. ${ }^{7}$ Earlier we have shown that surface related transitions dominate the low-energy part while bulk-related transitions are more pronounced in the high-energy part of the RAS spectra. ${ }^{7}$ The top-Ga dimer, mixed dimer, and $\delta$ structures show a pronounced negative anisotropy in the low-energy region, with minima between 2.0 and $2.3 \mathrm{eV}$. The strength of that anisotropy is directly correlated to the number of $\mathrm{Ga}-\mathrm{Ga}$ bonds along the [110] direction. Its magnitude is the highest for the top-Ga-dimer model with eight bonds, slightly reduced and shifted to lower energies for the mixed-dimer geometry with six cation-cation bonds and flattened for the $\delta$ structure with only two such bonds.

The positive anisotropy in the high energy region of the spectra is correlated to the formation of $\mathrm{P}-\mathrm{P}$ dimers oriented along [110]: For the $\beta 2$ geometry with three $\mathrm{P}-\mathrm{P}$ dimers, we find a relatively strong positive anisotropy between about 2.4 and $4.4 \mathrm{eV}$. The shape of that anisotropy is roughly preserved for the $\delta$ structure, featuring one $\mathrm{P}-\mathrm{P}$ dimer, however,
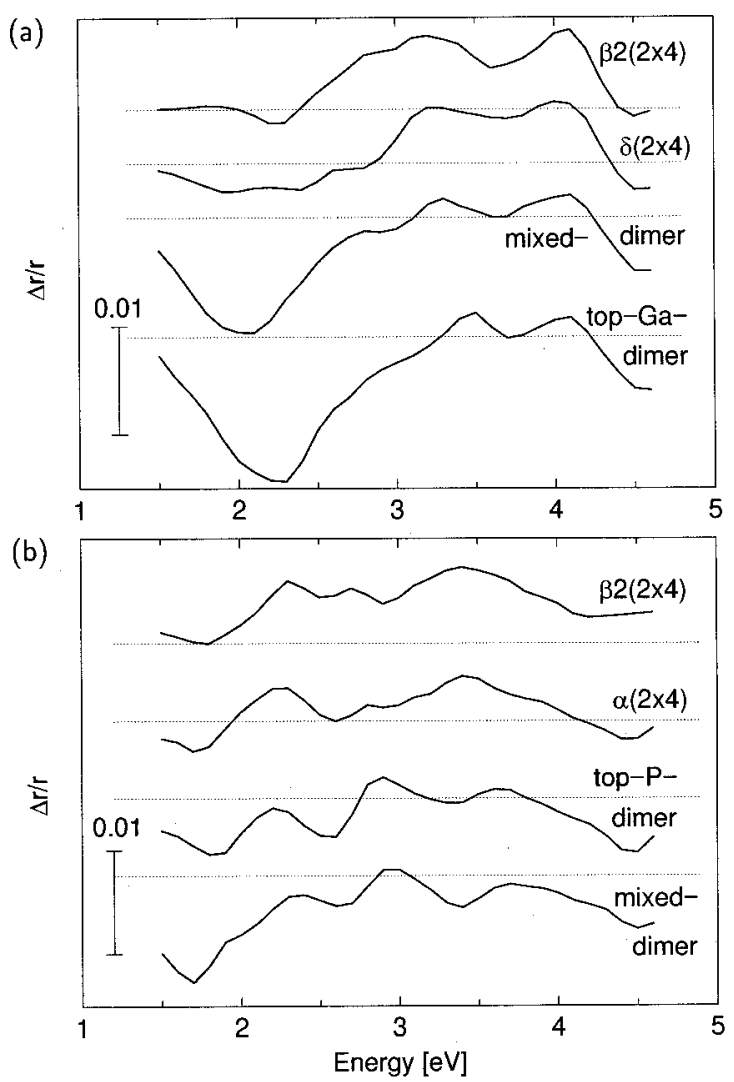

FIG. 5. (a) RAS spectra (same convention as in Fig. 2) calculated for the energetically favored structural models of the $\operatorname{GaP}(001)(2 \times 4)$ surface. The zero line in each spectrum is indicated by a horizontal dotted line. (b) Same as in (a), but for $\operatorname{InP}(001)$ surface structures.

with reduced magnitude and the spectrum is shifted down. An even further reduction in positive anisotropy occurs for the mixed-dimer and top-Ga-dimer structures (no P-P dimers).

According calculations for $\operatorname{InP}(001)^{6}$ are shown in the lower panel of Fig. 5. Similar systematic trends as for $\mathrm{GaP}(001)$ are observed in the optical anisotropy of $\operatorname{InP}(001)$ : cation-rich surfaces show a characteristic strong minimum at low energies, P-dimer terminated ones a broad positive anisotropy at higher energies.

In fact, apart from some energy shift due to the neglect of many-body effects in DFT-LDA, the calculated spectra are in rather good agreement with the experimental optical anisotropy. Together with the calculated phase diagrams they allow a conclusive comparison. The RAS spectra measured for the $(2 \times 4)$ structures at higher annealing temperature (In/Ga-rich phases) feature a strong negative peak in the lowenergy region. Given the energy position of this peak and its dependence on the preparation conditions, it can be identified with the calculated negative anisotropy arising from cationcation surface bond related states. Both the top-Ga-dimer model and the mixed-dimer model thus are plausible candidates to explain the Ga-rich surface phase on $\mathrm{GaP}(001)$, while on InP the mixed-dimer model represents the only possible structure under In-rich conditions.

The measured spectrum for the $\mathrm{GaP}(001)(2 \times 4)$ struc- 
ture annealed at lower temperature (more P-rich phase) is dominated by a "camelback" overall spectrum shape with maxima between the energies of the $E_{0}$ and $E_{1}$ critical points and at the $E_{0}^{\prime}$ bulk peak. No negative anisotropy appears. The only computed spectrum with no (or very little) negative anisotropy belongs to the $\beta 2(2 \times 4)$ structure. Maxima appear at 3.2 and $4.1 \mathrm{eV}$, close to the calculated energies of the $E_{1}$ and $E_{0}^{\prime}$ critical points. Our results thus indicate that the less Ga-rich phase of the $\mathrm{GaP}(001)(2 \times 4)$ surface corresponds to the $\beta 2(2 \times 4)$ structure known from As-rich $\mathrm{GaAs}(001)$ surfaces. This condition of a vanishing negative anisotropy is clearly not fulfilled in the case of the more P-rich InP(001) $(2 \times 4)$ structure. Consequently, this structure should be different, still containing In-In bonds in addition to $\mathrm{P}$ dimers. As mentioned above, a possible explanation would be a surface containing different domains.

\section{CONCLUSIONS}

In conclusion, we have presented a comprehensive study of the optical anisotropy of $\mathrm{GaP}(001)$ and $\mathrm{InP}(001)$ surfaces based on the comparison of experimental results with calculated surface geometries and reflectance anisotropy spectra. Both for a balanced surface stoichiometry and for cation-rich conditions, we find $(2 \times 4)$ reconstructed surfaces that are stabilized by the formation of dimers. Experiment as well as theory suggest the existence of different $(2 \times 4)$ surface phases, depending on the cation content of the surface. Our results indicate that mixed dimers on top of an cationterminated surface are the ground states of the $\mathrm{Ga} / \mathrm{In}$-rich phase of $\mathrm{GaP}(001)$ and $\mathrm{InP}(001)$ surfaces, consistent with previous findings for the $\operatorname{InP}(001)(2 \times 4)$. For the less cation-rich surface phases, we suggest the formation of $\mathrm{P}-\mathrm{P}$ dimers in a $\beta 2(2 \times 4)$ geometry for $\mathrm{GaP}(001)$. In case of InP, however, cation-cation bonds seem to be still present at the surface.

\section{ACKNOWLEDGMENTS}

Financial support by BMBF-Verbundprojekt 22, DFG (Schm 1361/1-1, Es 127/4-1), NSF (DMR 9408437), and ONR (N00014-96-I-0161) is gratefully acknowledged. This work was supported in part by grants of supercomputer time from the DoD Challenge Program and the North Carolina Supercomputer Center.

${ }^{1}$ Q.-K. Xue, T. Hashizume, and T. Sakurai, Prog. Surf. Sci. 56, 1 (1997), and references therein.

${ }^{2}$ W. Richter and J.-T. Zettler, Appl. Surf. Sci. 100/101, 465 (1996).

${ }^{3}$ I. Kamiya, D. E. Aspnes, L. T. Florez, and J. P. Harbison, Phys. Rev. B 46, 15894 (1992).

${ }^{4}$ M. Murayama, K. Shiraishi, and T. Nakayama, Jpn. J. Appl. Phys., Part 1 37, 4109 (1998).

${ }^{5}$ A. I. Shkrebtii, N. Esser, W. Richter, W. G. Schmidt, F. Bechstedt, B. O. Fimland, A. Kley, and R. Del Sole, Phys. Rev. Lett. 81, 721 (1998).
${ }^{6}$ W. G. Schmidt, E. Briggs, J. Bernholc, and F. Bechstedt, Phys. Rev. B 59, 2234 (1999).

${ }^{7}$ A. M. Frisch, W. G. Schmidt, J. Bernholc, M. Pristovsek, N. Esser, and W. Richter, Phys. Rev. B (to be published).

${ }^{8}$ W. G. Schmidt, F. Bechstedt, N. Esser, M. Pristovsek, Ch. Schultz, and W. Richter, Phys. Rev. B 57, 14596 (1998); W. G. Schmidt and F. Bechstedt, Surf. Sci. 409, 474 (1998).

${ }^{9}$ D. K. Biegelsen, R. D. Bringans, J. E. Northrup, and L.-E. Swartz, Phys. Rev. 41, 5701 (1990).

${ }^{10}$ J. E. Northrup and S. Froyen, Mater. Sci. Eng., B 30, 81 (1995).

${ }^{11}$ W. G. Schmidt, Appl. Phys. A: Mater. Sci. Process. 65, 581 (1997).

${ }^{12}$ J. Kinsky, Ch. Schultz, D. Pahlke, A. M. Frisch, T. Herrmann, N. Esser, and W. Richter, Appl. Surf. Sci. 123, 228 (1998).

${ }^{13}$ C. D. MacPherson, R. A. Wolkow, C. E. J. Mitchell, and A. B. McLean, Phys. Rev. Lett. 77, 691 (1996).

${ }^{14}$ M. M. Sung, C. Kim, H. Bu, D. S. Karpuzov, and J. W. Rabalais, Surf. Sci. 322, 116 (1995).

${ }^{15}$ Y. Fukuda, M. Shimomura, N. Sanada, and N. Nagoshi, J. Appl. Phys. 76, 3632 (1994)

${ }^{16}$ N. Sanada, M. Shimomura, and Y. Fukuda, and T. Sato, Appl. Phys. Lett. 67, 1432 (1995)

${ }^{17}$ A. Watanabe, H. Shimaya, M. Naitoh, and S. Nishigaki, J. Vac. Sci. Technol. B 14, 3599 (1996).

${ }^{18}$ M. Naitoh, A. Watanabe, A. Konishi, and S. Nishigaki, Jpn. J. Appl. Phys., Part 1 35, 4789 (1996).

${ }^{19}$ M. M. Sung and J. W. Rabalais, Surf. Sci. 365, 136 (1996).

${ }^{20}$ N. Oishi, F. Shoji, A. Konishi, M. Naitoh, and S. Nishigaki, Surf. Rev. Lett. 5, 223 (1998).

${ }^{21}$ I. M. Vitomirov, A. Raisanen, L. J. Brillson, C. L. Lin, D. T. McInturff, P. D. Kirchner, and J. M. Woodall, J. Vac. Sci. Technol. A 11, 841 (1993).

${ }^{22}$ M. Naitoh, A. Konishi, H. Inenaga, S. Nishigaki, N. Oishi, and F. Shoji, Surf. Sci. 402-404, 623 (1998).

${ }^{23}$ J. N. Baillargeon, K. Y. Cheng, and K. C. Hsieh, Appl. Phys. Lett. 56, 2201 (1990).

${ }^{24}$ M. Yoshikawa, A. Nakamura, T. Nomura, and K. Ishikawa, Jpn. J. Appl. Phys., Part 1 35, 1205 (1995).

${ }^{25}$ P. A. Postigo, G. Armelles, and F. Briones, Phys. Rev. B 58, 9659 (1998).

${ }^{26}$ J. S. Luo, J. F. Geisz, J. M. Olson, and M. C. Wu, J. Cryst. Growth 174, 558 (1997).

${ }^{27}$ K. Knorr, M. Pristovsek, U. Resch-Esser, N. Esser, M. Zorn, and W. Richter, J. Cryst. Growth 170, 230 (1997).

${ }^{28}$ M. Zorn, B. Junno, T. Trepk, S. Bose, L. Samuelson, J.-T. Zettler, and W. Richter, Phys. Rev. B (submitted).

${ }^{29}$ (a) N. Esser, U. Resch-Esser, M. Pristovsek, and W. Richter, Phys. Rev. B 53, R13257 (1996); (b) Th. Hannappel, S. Visbeck, K. Knorr, J. Mahrt, M. Zorn, and F. Willig, Appl Phys. A: Mater. Sci. Process. (in press).

${ }^{30}$ R. Del Sole, Solid State Commun. 37, 537 (1981).

${ }^{31}$ F. Manghi, R. Del Sole, A. Selloni, and E. Molinari, Phys. Rev. B 41, 9935 (1990).

${ }^{32}$ S. Zollner, M. Garriga, J. Kirchner, J. Humliicek, and M. Cardona, Phys. Rev. B 48, 11 (1993).

${ }^{33}$ P. Lautenschlager, M. Garriga, and M. Cardona, Phys. Rev. B 36, 4813 (1987).

${ }^{34}$ K. B. Ozanyan, P. J. Parbrook, M. Hopkinson, C. R. Whitehouse, Z. Sobiesierski, and D. I. Westwood, J. Appl. Phys. 82, 474 (1997).

${ }^{35}$ P. Vogt, Th. Hannappel, S. Visbeck, K. Knorr, N. Esser, and W. Richter, Phys. Rev. B (to be published).

${ }^{36}$ W. G. Schmidt and F. Bechstedt, Phys. Rev. B 55, 13051 (1997).

${ }^{37}$ W. G. Schmidt and F. Bechstedt, Phys. Rev. B 54, 16742 (1996).

${ }^{38}$ M. D. Pashley, Phys. Rev. B 40, 10481 (1989).

${ }^{39}$ M. Zorn, T. Trepk, J.-T. Zettler, B. Junno, C. Meyne, K. Knorr, T. Wethkamp, M. Klein, M. Miller, W. Richter, and L. Samuelson, Appl. Phys. A: Mater. Sci. Process. 65, 333 (1997). 